\title{
Donor Complications Following Laparoscopic Compared to Hand-Assisted Living Donor Nephrectomy: An Analysis of the Literature
}

\author{
Whitney R. Halgrimson, ${ }^{1}$ Jeffrey Campsen, ${ }^{1}$ M. Susan Mandell, ${ }^{1,2}$ Mara A. Kelly, ${ }^{1}$ \\ Igal Kam, ${ }^{1}$ and Michael A. Zimmerman ${ }^{1}$ \\ ${ }^{1}$ Division of Transplant Surgery, University of Colorado Health Sciences Center, P.O. Box 6510, Aurora, CO 80045I, USA \\ ${ }^{2}$ Department of Anesthesia, University of Colorado Health Sciences Center, P.O. Box 6510, Aurora, CO 80045I, USA
}

Correspondence should be addressed to Michael A. Zimmerman, michael.zimmerman@ucdenver.edu

Received 10 August 2009; Accepted 2 November 2009

Academic Editor: Bruce Kaplan

Copyright ( $) 2010$ Whitney R. Halgrimson et al. This is an open access article distributed under the Creative Commons Attribution License, which permits unrestricted use, distribution, and reproduction in any medium, provided the original work is properly cited.

\begin{abstract}
There are two approaches to laparoscopic donor nephrectomy: standard laparoscopic donor nephrectomy (LDN) and handassisted laparoscopic donor nephrectomy (HALDN). In this study we report the operative statistics and donor complications associated with LDN and HALDN from large-center peer-reviewed publications. Methods. We conducted PubMed and Ovid searches to identify LDN and HALDN outcome studies that were published after 2004. Results. There were 37 peer-reviewed studies, each with more than 150 patients. Cumulatively, over 9000 patients were included in this study. LDN donors experienced a higher rate of intraoperative complications than HALDN donors $(5.2 \%$ versus. $2.0 \%, P<.001)$. Investigators did not report a significant difference in the rate of major postoperative complications between the two groups (LDN $0.5 \%$ versus HALDN $0.7 \%$, $P=.111)$. However, conversion to open procedures from vascular injury was reported more frequently in LDN procedures $(0.8 \%$ versus $0.4 \%, P=.047)$. Conclusion. At present there is no evidence to support the use of one laparoscopic approach in preference to the other. There are trends in the data suggesting that intraoperative injuries are more common in LDN while minor postoperative complications are more common in HALDN.
\end{abstract}

\section{Introduction}

There has been a rapid increase in living organ donation in order to bridge the shortfall between the demand and supply of donor organs. Kidney transplant recipients have benefited considerably by the expansion in living donation. The 2007 annual report from the Scientific Registry of Transplant Recipients (SRTR) shows that $45 \%$ of all transplanted kidneys were obtained from living donors [1]. Until 1995, donor nephrectomy surgery was performed through a large flank incision. Subsequently, laparoscopic donor nephrectomy (LDN) became the surgical approach of choice [2].

Evidence shows that LDN does not adversely affect donor graft function or survival compared to kidney recovery through an open approach [3]. Laparoscopic techniques also reduce length of hospitalization, improve pain scores, and produce a better cosmetic outcome [4-7]. Nonetheless, LDN requires additional technical training with a distinct learning curve, and surgeons may initially experience longer operating and warm ischemic times $[5,8,9]$. Published reports also suggest that $\mathrm{LDN}$ is more technically challenging than open donor nephrectomy (ODN) in patients who are greater than their ideal body weight [2].

Surgeons have modified the technique of $\mathrm{LDN}$ in order to make the recovery of the donor kidney easier and safer. One approach uses a longer incision at the extraction site so that the surgeon's hand can be inserted into the peritoneal cavity to assist with laparoscopic donor nephrectomy $[10,11]$. Some investigators report shorter warm ischemic times and less intraoperative bleeding with hand-assisted laparoscopic nephrectomy (HALDN) compared to LDN [12]. HALDN 
has also been used in donors who were previously considered poor candidates for LDN, including those who weigh more than their ideal body weight, who have anatomic variations in the renal vasculature or who had previous abdominal surgery [12]. Generally the rate of complications appears to be similar between HALN and LDN. However, the type and severity of complications has not been systematically compared.

Currently the choice of procedure largely depends on the personal preference and experience of the surgeon $[2,13]$. A 2008 survey of transplant fellowship training programs reported that $41 \%$ of responding centers preferred $\mathrm{LDN}$, $22 \%$ preferred HALDN, and $37 \%$ used a combination of LDN and HALDN procedures [2]. The variability in practice can be partly attributed to a lack of detail about the risks associated with both techniques [12]. In this study we report summarized information on the rate and type of donor complications derived from large retro- and prospective institutional publications on LDN and HALDN.

\section{Methods}

2.1. Literature Search. We performed Pubmed and Ovid keyword searches to identify all studies on LDN and HALDN. Search words included laparoscopic donor nephrectomy, LDN, and HALDN. We included all literature that was published between January 01, 2004 and April 30, 2009. The inclusion criteria for our review were studies that enlisted adult patients, only, described a transperitoneal surgical technique, and were published in English. Studies were included that described outcomes in greater than 150 LDN patients or 50 HALDN patients or studies that directly compared outcomes between the two groups in any number of patients. A total of 37 studies were included in the final analysis. A total of 206 studies were excluded because they did not use standard laparoscopic or hand-assisted approaches (e.g., retroperitoneal approach, robot-assistance, etc.), they were review or editorial publications, or they did not contain specific information on donor outcomes. In cases where one study population was contained in more than one publication, we analyzed the most recently published report that included the largest study cohort and that focused on donor outcomes.

2.2. Data Collection. We were unable to construct a standard metadatabank for analysis because the design of all the studies uncovered by the search was descriptive. Therefore studies lacked standard techniques for validity assessment and data abstraction such as blinding, reliability checks, or duplicate observations. In addition, we accepted more than one type of data collection method, including retrospective, prospective, and randomized controlled. We therefore could not use meta-analysis and chose instead to summate the data in order to provide an estimate of the risks and complications associated with LDN compared to HALDN.

We summated the data for the categories of LDN and for HALDN and recorded eight distinct patient and outcome variables. These included age, body mass index (BMI), total operative time, estimated blood loss, warm ischemic time, length of hospital stay, intraoperative and postoperative complications. Deaths during the admission hospitalization were also recorded.

2.3. Data Analysis. Discrete variables were recorded as the number of reported events, while continuous measures (age, BMI, total donor operative time, estimated blood loss, warm ischemia time, length of hospital stay) were expressed as the weighted mean of the reported arithmetic means in the dataset. Weighted mean calculations were based on sample size due to the lack of reporting of the standard error from many studies. In studies that did not explicitly define their measure of total donor operative time we used a measure from skin incision to skin closure. Twenty of the 34 studies that reported total operative time did not give a specific definition, and three studies were excluded from the calculation of operative time because they used alternative endpoints in their definition. Similarly, we estimated warm ischemia time (WIT) from renal vessel occlusion to immersion in ice or back-table perfusion if this information was not included. Seventeen of 40 studies reporting WIT did not give an explicit definition but did provide enough information to estimate the WIT using the above definition.

We identified complications that represented significant donor morbidity or mortality and led to an escalation in care. These were blood transfusion, aborted procedure, conversion to open surgical procedure, rehospitalization, reoperation, and death. Complication rates were compared using a two-tailed $\mathrm{z}$ test of two binomial proportions. The calculated weighted means were compared using the "glm" procedure function in SAS software.

\section{Results}

3.1. Database. The search produced 37 studies that met all the inclusion criteria. Nine of the 37 studies reported and compared outcomes of both HALDN and LDN. Twelve described the outcomes of only HALDN outcomes and 16 described only LDN outcomes. There were 26 retrospective, eight prospective, and one randomized control trial. Two studies did not clearly define their study design. The dataset derived from the 37 publications included 9296 patients, of which 2562 were HALDN and 6734 were LDN. Details of the study design are reported in Table 1 . The intraoperative and post-operative donor complications were classified into categories based on the organ system that was injured and if the injury was related to procedural problems. The classification of the intra and post-operative complications is shown in Tables 2 and 3.

Patient demographics and intraoperative weighted mean values are displayed in Table 1, although we could not extract complete information in all the analytic categories. Of the 37 studies, 34 reported total operative time, 22 reported estimated blood loss, 30 reported WIT, and 29 reported length of stay. In addition, the BMI of donor nephrectomy patients was only reported in 18 studies, making it the least reported variable.

The average age of LDN donors was 39.9 years while that of the HALDN donors was 41.2 years. Donors had similar 
TABle 1: Study characteristics, donor demographics and intraoperative data.

\begin{tabular}{|c|c|c|c|c|c|c|c|c|c|c|c|c|c|c|c|}
\hline \multirow[t]{2}{*}{ Author et al. } & \multirow[t]{2}{*}{$\begin{array}{l}\text { Study } \\
\text { type }\end{array}$} & \multicolumn{2}{|c|}{ Cases } & \multicolumn{2}{|c|}{$\begin{array}{c}\text { Age } \\
\text { (years) }\end{array}$} & \multicolumn{2}{|c|}{$\begin{array}{c}\text { BMI } \\
(\mathrm{kg} / \mathrm{m} 2)\end{array}$} & \multicolumn{2}{|c|}{$\begin{array}{c}\text { Total } \\
\text { operative } \\
\text { time } \\
(\mathrm{min})\end{array}$} & \multicolumn{2}{|c|}{$\begin{array}{c}\text { Operative } \\
\text { blood loss } \\
(\mathrm{mL})\end{array}$} & \multicolumn{2}{|c|}{$\begin{array}{c}\text { Warm } \\
\text { ischemia } \\
\text { time } \\
\text { (min) }\end{array}$} & \multicolumn{2}{|c|}{$\begin{array}{c}\text { Length of } \\
\text { stay } \\
\text { (days) }\end{array}$} \\
\hline & & $\mathrm{L}$ & $\mathrm{H}$ & $\mathrm{L}$ & $\mathrm{H}$ & $\mathrm{L}$ & $\mathrm{H}$ & $\mathrm{L}$ & $\mathrm{H}$ & $\mathrm{L}$ & $\mathrm{H}$ & $\mathrm{L}$ & $\mathrm{H}$ & $\mathrm{L}$ & $\mathrm{H}$ \\
\hline Anderson [14] & $\mathrm{R}$ & & 103 & & 34.4 & & 25.9 & & 283 & & & & 2.3 & & 3.7 \\
\hline Bargman [15] & RCT & 20 & 20 & & & & & 200 & 219 & 141.5 & 97.4 & 2.6 & 2.3 & 1.9 & 2.1 \\
\hline Branco [16] & $\mathrm{R}$ & 89 & 67 & 38.9 & 38 & & & 78.4 & 83 & 98.9 & 130.9 & 2.5 & 3.6 & 1.4 & 2.8 \\
\hline Breda [17] & $\mathrm{R}$ & 300 & & 36.7 & & 28.3 & & 180 & & 80 & & 4.0 & & & \\
\hline Chandak [18] & $\mathrm{P}$ & & 144 & & 44 & & & & 198 & & 160 & & 2.9 & & \\
\hline Chin [9] & $\mathrm{R}$ & 500 & & 40.3 & & 27.3 & & 208.2 & & 197 & & 3.5 & & 2.3 & \\
\hline Desai [19] & & 303 & & 45.2 & & 24.2 & & 159.0 & & & & 6.5 & & 4.4 & \\
\hline Diner [20] & $\mathrm{R}$ & 167 & & 39.0 & & & & 226.3 & & 108.3 & & 5.0 & & 2.5 & \\
\hline Dolce [21] & $\mathrm{P}$ & & 217 & & 39.6 & & & & 177.3 & & 71.1 & & 1.1 & & 3.6 \\
\hline Dols [22] & $\mathrm{P}$ & 283 & & 50 & & & & 222 & & 206 & & 5.6 & & 3.5 & \\
\hline El-Galley [23] & $\mathrm{P}$ & & 80 & & 43 & & & & 184 & & 50 & & 4.0 & & \\
\hline Fettouh [24] & $\mathrm{R}$ & 400 & & 32 & & & & $117^{*}$ & & 56 & & 2.6 & & & \\
\hline Fisher [25] & $\mathrm{R}$ & & 200 & & 42 & & 27 & & 229 & & 243 & & 2.6 & & 1.9 \\
\hline Giron [26] & $\mathrm{R}$ & & 85 & & 34.3 & & & & 132 & & 125 & & 4.0 & & 2.5 \\
\hline Gupta [27] & $\mathrm{P}$ & 343 & & 43 & & & & 191 & & & & 3.3 & & 3.7 & \\
\hline Hawasli [28] & $\mathrm{R}$ & 168 & & & & & & 132 & & 92 & & 3.5 & & 1.2 & \\
\hline Heimbach [29] & $\mathrm{R}$ & & 553 & & 42 & & 28.0 & & $137^{*}$ & & & & & & 2.3 \\
\hline Husted [30] & $\mathrm{R}$ & 213 & 40 & 41 & 41 & 27.5 & & 192 & 186 & & & 3.3 & 3.9 & 1.9 & 1.9 \\
\hline Jacobs [31] & $\mathrm{R}$ & 738 & & 40.2 & & 27.7 & & 202.1 & & 128 & & 2.8 & & 2.7 & \\
\hline Jeon [32] & $\mathrm{R}$ & 71 & 12 & 36 & 38 & & & $206.7^{*}$ & $143.4^{*}$ & & & 3.8 & 2.4 & & \\
\hline Keller [33] & $\mathrm{R}$ & & 230 & & & & & & 176 & & 71 & & 1.1 & & 3.7 \\
\hline Ko [34] & $\mathrm{R}$ & 400 & & 40.9 & & 27.1 & & 147.6 & & 83.2 & & 1.5 & & 2.0 & \\
\hline Kocak [35] & $\mathrm{R}$ & 482 & 318 & 39 & 41 & 27 & 29 & & & & & & & 1.6 & 1.2 \\
\hline Lai [36] & $\mathrm{P}$ & 12 & 12 & 48.6 & 44 & 22.5 & 25 & 215 & 258 & & & 4.5 & 3.8 & 5.8 & 5.6 \\
\hline Lallas [37] & $\mathrm{R}$ & 230 & & 40.6 & & & & 112 & & 92.8 & & & & 2.3 & \\
\hline $\operatorname{Li}[38]$ & $\mathrm{R}$ & & 65 & & & & & & 157.92 & & 37.38 & & 3.3 & & 5.0 \\
\hline Melcher [39] & $\mathrm{R}$ & 530 & & 40.4 & & 26.1 & & 196 & & & & & & 3.2 & \\
\hline Minnee [40] & $\mathrm{P}$ & & 158 & & 46.7 & & & & 173.8 & & & & 3.2 & & 4.9 \\
\hline Percegona [41] & $\mathrm{R}$ & 34 & 21 & & & & & 184 & 191 & 441 & 545 & 3.8 & 4.3 & 2.6 & 3.6 \\
\hline Permpongkosol [42] & $\mathrm{R}$ & 553 & & 41.4 & & & & & & & & & & & \\
\hline Rajab $[43]$ & $\mathrm{R}$ & & 80 & & 38 & & 26.5 & & & & & & & & \\
\hline Ruszat [44] & $\mathrm{R}$ & 12 & 33 & 46 & 50 & 24 & 24 & 212 & 192 & 307 & 208 & 4.0 & 2.1 & 13.0 & 11.0 \\
\hline Salazar [45] & & 11 & 24 & 39 & 44 & 26 & 26 & 213 & 235 & & & & & 3.0 & 4.0 \\
\hline Seo $[46]$ & $\mathrm{R}$ & & 100 & & 38.1 & & 23 & & 202 & & & & 3.0 & & 4.1 \\
\hline Simforoosh [47] & $\mathrm{P}$ & 241 & & 27.8 & & 24.8 & & 136.5 & & & & 7.5 & & & \\
\hline $\mathrm{Su}[48]$ & $\mathrm{R}$ & 381 & & & & & & 253 & & 334 & & 4.9 & & 3.3 & \\
\hline Sundaram [49] & $\mathrm{R}$ & 253 & & 39.3 & & 26.1 & & 199 & & 115 & & 2.2 & & 2.8 & \\
\hline Total & & 6734 & 2562 & & & & & & & & & & & & \\
\hline Weighted mear & & & & 39.9 & 41.2 & 26.8 & 27.3 & 187.0 & 189.3 & 144.0 & 128.6 & 3.7 & 2.5 & 2.7 & 3.0 \\
\hline Difference (L-H) (95 & $5 \% \mathrm{CI})$ & & & -1.3( & $4.2,1.7)$ & -0.5( & $1.8,0.8)$ & $-2.3(-$ & $1.4,26.7)$ & $15.4(-$ & $5.1,95.8)$ & $1.2(0$ & $1,2.3)$ & -0.3 & $-1.1,0.6$ \\
\hline$P$-value & & & & & 401 & & 26 & & 76 & & 711 & & $37^{\dagger}$ & & 547 \\
\hline
\end{tabular}

R:Retrospective; P:Prospective; RCT:Randomized Control Trial; L:LDN; H:HALDN.

* Excluded from weighted mean calculation (alternative endpoints); ${ }^{\dagger} P<.05$. 
TABLE 2: Intraoperative complications of LDN and HALDN

\begin{tabular}{|c|c|c|c|c|c|}
\hline \multirow{2}{*}{ Bowel injury } & \multicolumn{2}{|c|}{$\mathrm{LDN}$} & \multicolumn{2}{|c|}{ HALDN } & \multirow{2}{*}{$\frac{P \text {-value }}{.031^{\dagger}}$} \\
\hline & 27 & $0.4 \%$ & 3 & $0.1 \%$ & \\
\hline Liver/mesenteric/splenic injury & 48 & $0.7 \%$ & 4 & $0.2 \%$ & $.001^{\dagger}$ \\
\hline \multicolumn{6}{|l|}{ Pulmonary injury } \\
\hline Atelectasis & 2 & $<0.0 \%$ & 0 & $0.0 \%$ & .383 \\
\hline Diaphragm injury & 4 & $0.1 \%$ & 0 & $0.0 \%$ & .217 \\
\hline \multirow[t]{2}{*}{ Pneumothorax } & 10 & $0.1 \%$ & 0 & $0.0 \%$ & .051 \\
\hline & 16 & $0.2 \%$ & 0 & $0.0 \%$ & $.014^{\dagger}$ \\
\hline \multicolumn{6}{|l|}{ Renal/ureteral injury } \\
\hline Bladder injury & 5 & $0.1 \%$ & 1 & $<0.0 \%$ & .550 \\
\hline Degloving of the kidney capsule & 2 & $<0.0 \%$ & 2 & $0.1 \%$ & .315 \\
\hline Renal laceration & 9 & $0.1 \%$ & 0 & $0.0 \%$ & .064 \\
\hline \multirow[t]{2}{*}{ Ureteral injury } & 10 & $0.1 \%$ & 2 & $0.1 \%$ & .398 \\
\hline & 26 & $0.4 \%$ & 5 & $0.2 \%$ & .154 \\
\hline \multicolumn{6}{|l|}{ Vascular injury/bleeding } \\
\hline Bleeding-vascular injury & 97 & $1.4 \%$ & 24 & $0.9 \%$ & .056 \\
\hline \multirow[t]{2}{*}{ Major bleeding (> 2 UPRBCs or $>500 \mathrm{~mL}$ ) } & 35 & $0.5 \%$ & 3 & $0.1 \%$ & $.007^{\dagger}$ \\
\hline & 132 & $2.0 \%$ & 27 & $1.1 \%$ & $.003^{\dagger}$ \\
\hline \multicolumn{6}{|l|}{ Procedural complications } \\
\hline Conversion-Elective/obesity & 17 & $0.3 \%$ & 4 & $0.2 \%$ & .382 \\
\hline Conversion—reason not reported/other & 19 & $0.3 \%$ & 5 & $0.2 \%$ & .460 \\
\hline Difficult manual extractions/entrapment sack malfunction & 12 & $0.2 \%$ & 0 & $0.0 \%$ & $.033^{\dagger}$ \\
\hline Endotracheal intubation/extubation difficulties & 3 & $<0.0 \%$ & 0 & $0.0 \%$ & .285 \\
\hline \multirow[t]{2}{*}{ Staple misfire } & 4 & $0.1 \%$ & 0 & $0.0 \%$ & .217 \\
\hline & 55 & $0.8 \%$ & 9 & $0.4 \%$ & $.015^{\dagger}$ \\
\hline \multicolumn{6}{|l|}{ Other complications } \\
\hline Cardiac arrhythmias & 2 & $<0.0 \%$ & 0 & $0.0 \%$ & .383 \\
\hline Hydrocele & 2 & $<0.0 \%$ & 0 & $0.0 \%$ & .383 \\
\hline Transient CO2 pneumoperitoneum & 2 & $<0.0 \%$ & 0 & $0.0 \%$ & .383 \\
\hline \multirow[t]{2}{*}{ Other } & 40 & $0.6 \%$ & 2 & $0.1 \%$ & $.001^{\dagger}$ \\
\hline & 46 & $0.7 \%$ & 2 & $0.1 \%$ & $<.001^{\dagger}$ \\
\hline Total intraoperative complications & 350 & $5.2 \%$ & 50 & $2.0 \%$ & $<.001^{\dagger}$ \\
\hline${ }^{\dagger} P<.05$ & & & & & \\
\hline
\end{tabular}

average BMI (26.8 kg/m² LDN versus $27.3 \mathrm{~kg} / \mathrm{m}^{2}$ HALDN). Mean operative time was 187.0 minutes in LDN procedures and 189.3 minutes in HALDN procedures. Mean length of stay was slightly shorter but not significantly different for LDN donors (2.7 days versus 3.0 days). Conversely, HALDN donor warm ischemic time was shorter (3.7 minutes versus 2.5 minutes) and estimated blood loss was less $(144.0 \mathrm{~mL}$ versus $128.6 \mathrm{~mL}$ ). However, only WIT was found to be significantly different between LDN and HALDN cases $(P=$ .037).

3.2. Intraoperative Complications. The rate of intraoperative complications was significantly higher in LDN donors compared to HALDN donors $(5.2 \%$ versus $2.0 \%, P<.001)$.
Vascular injury or bleeding, bowel injury, pulmonary injury, and liver, mesenteric or splenic injury were significantly more common with LDN (Table 2). There was also a greater rate of technical difficulties encountered during surgery with LDN. We did not find a significant difference in the incidence of renal or ureteral injury.

The LDN rate of vascular injury or bleeding was $2.0 \%$ while the HALDN rate was only $1.1 \%(P=.003)$. Within the LDN group there was also a higher rate of major bleeding (defined as greater than two units of packed red blood cells or $500 \mathrm{~mL}$ blood loss). The rate of bleeding due to other vascular injuries was also elevated in LDN donors, but we did not find a statistically significant difference when compared with HALDN (1.4\% versus $0.9 \%, P=.056)$. LDN donors 
also experienced significantly higher rates of bowel injury ( $0.4 \%$ versus $0.1 \%, P=.031)$, and liver, mesenteric or splenic injury $(0.7 \%$ versus $0.2 \%, P=.001)$.

Procedural complications occurred more frequently in LDN than in HALDN cases $(0.8 \%$ versus $0.4 \%$, $P=.008)$. Most complications were reported as difficult manual extractions, entrapment sack malfunctions, or elective conversions to open procedures. Only difficult manual extractions and entrapment sack malfunctions were found to be significantly elevated $(0.2 \%$ versus $0.0 \%, P=.033)$.

3.3. Postoperative Complications. The incidence of postoperative complications in LDN donors was statistically lower than in HALDN donors $(6.7 \%$ versus $8.6 \%, P=$ .002) (Table 3). There was no statistical difference found between the summated rates of cardiac, neural, pulmonary, or vascular complications. However, we observed striking differences between LDN and HALDN rates of incisional, renal, and ureteric complications. Incision and wound complications occurred in $1.7 \%$ of LDN donors compared to $3.1 \%$ of HALDN donors $(P<.001)$. Notably, LDN had lower rates of incisional hernias $(0.1 \%$ versus $0.7 \%, P<.001)$ and wound infections $(1.4 \%$ versus $2.0 \%, P=.034)$. The rates of abdominal and bowel complications were also found to be significantly different. There were no statistical differences in other complications listed in this category.

LDN donors had a markedly lower incidence of renal and ureteric complications $(0.4 \%$ versus $1.6 \%, P<.001)$. This finding was explained by the larger number of HALDN patients who suffered from urinary retention and urinary tract infections (both $0.2 \% \mathrm{LDN}$ versus $0.7 \%$ HALDN, $P<.001)$. The rate of post-operative ileus was significantly greater in HALDN donors than in LDN donors $(0.5 \%$ versus $1.0 \%, P=.008)$. This elevated ileus rate was the primary determinant of overall bowel complications in HALDN groups $(0.7 \%$ versus $1.1 \%, P=.025)$.

The LDN and HALDN donors experienced similar rates of post-operative vascular complications and hematomas, $0.9 \%$ versus $0.7 \%$, respectively $(P=.525)$. However, there were significant differences within this category. The rates of subcapsular hematoma and continued bleeding were statistically greater in the LDN donors $(P=.026)$. Conversely, only HALDN donors experienced pulmonary embolism or deep vein thrombus $(0.0 \%$ versus $0.2 \%, P=$ $.001)$.

3.4. Major Complications. The summated rate of major intraoperative complications was significantly higher in LDN donors than in HALDN donors $(2.2 \%$ versus $1.1 \%, P=$ $.001)$. This difference was due to major injuries that required blood transfusions or conversion to an open procedure. Blood transfusions were needed in $0.8 \%$ of LDN donors versus only $0.2 \%$ of HALDN donors $(P=.004)$. The overall rate of conversions to open surgery was also significantly higher in LDN patients $(1.3 \%$ versus $0.8 \%, P=.030)$, primarily due to bleeding $(0.8 \%$ versus $0.4 \%, P=.047)$.

We did not find a significant difference in the rate of conversions to an open procedure due to obesity or other reasons. Even though there were two aborted procedures in the LDN group, due to a colon and a mesenteric vein injury, no significant difference was detected between the two groups.

The rate of major post-operative complications was similar in HALDN donors ( $0.5 \%$ versus $0.7 \%, P=.111$ ). Significant differences were only observed in a higher incidence of rehospitalization among HALDN donors $(0.3 \%)$ versus LDN donors $(0.1 \%)(P=.007)$. Ileus was the most common reported reason for rehospitalization. Reoperations were needed in $0.4 \%$ of both types of laparoscopic procedure $(P=$ $.807)$. The indications for additional surgery included bleeding or hematoma, bowel injury, small bowel obstruction, and incisional hernias. Two patients died perioperatively: one HALDN donor died due to a thromboembolism and one LDN donor died due to a myocardial infarction.

The overall rate of major intraoperative and postoperative complications was $2.6 \%$ in LDN donors and was $1.8 \%$ in HALDN donors. The difference was found to be statistically significant $(P=.013)$. The relative rates of all major complications are found in Table 4 .

\section{Discussion}

Living donor nephrectomy is unique in that the donor assumes an operative risk yet has no underlying disease and does not have any direct medical benefit from the procedure. This explains the clear imperative to ensure that donor complications are minimized $[3,40]$. While LDN and HALDN techniques have been shown to be safe compared to open surgery, both techniques appear to have different rates and types of complications. The data we analyzed suggest that LDN donors experience higher rates of intraoperative complications than HALDN donors. Furthermore, the incidence of major bleeding and overall vascular injury was also greater in the LDN group and more commonly required open conversion and blood transfusions. Conversely, the rate of postoperative wound infections renal and ureteric injuries appeared to be greater in the HALDN group.

While proponents of HALDN point out the advantages of a kidney extraction site and improved tactile control during the procedure, investigators have questioned if HALDN patients suffer from a greater number of post-operative complications [50]. Our data analysis strongly supports the impression that HALDN donors have greater incision morbidity than LDN donors. This contrasts with the findings of Kocak et al., who reported no significant differences in incision morbidity in a large direct comparison of LDN and HALDN donor complications [35]. We have no data to explain this discrepancy, but it may suggest that institutional practices and technical experiences play a role in determining outcomes unique to each study. We did not find significant differences in the rate of re-hospitalization due to infection nor the rate of re-operation for incisional hernia.

Post-operative bowel complications were cited as significant sources of donor morbidity in HALDN and thus are the reason why some centers choose to employ the LDN technique in favor of HALDN [35]. We identified greater 
TABLE 3: Postoperative complications of LDN and HALDN.

\begin{tabular}{|c|c|c|c|c|c|}
\hline & \multicolumn{2}{|c|}{ LDN } & \multicolumn{2}{|c|}{ HALDN } & \multirow[t]{2}{*}{$P$-value } \\
\hline Abdominal complications & & & & & \\
\hline Chylous ascites & 5 & $0.1 \%$ & 4 & $0.2 \%$ & .257 \\
\hline Other & 10 & $0.1 \%$ & 11 & $0.4 \%$ & $.011^{\dagger}$ \\
\hline & 15 & $0.2 \%$ & 15 & $0.6 \%$ & $.006^{\dagger}$ \\
\hline \multicolumn{6}{|l|}{ Bowel complications } \\
\hline Ileus & 35 & $0.5 \%$ & 26 & $1.0 \%$ & $.008^{\dagger}$ \\
\hline Intestinal obstruction & 9 & $0.1 \%$ & 2 & $0.1 \%$ & .486 \\
\hline \multirow[t]{2}{*}{ Other } & 1 & $<0.1 \%$ & 1 & $<0.1 \%$ & .478 \\
\hline & 45 & $0.7 \%$ & 29 & $1.1 \%$ & $.025^{\dagger}$ \\
\hline \multicolumn{6}{|l|}{ Cardiac complications } \\
\hline Hypertension requiring medication & 11 & $0.2 \%$ & 0 & $0.0 \%$ & $.041^{\dagger}$ \\
\hline Myocardial infarction & 1 & $<0.0 \%$ & 0 & $0.0 \%$ & .537 \\
\hline \multirow[t]{2}{*}{ Other } & 2 & $<0.1 \%$ & 1 & $<0.1 \%$ & .823 \\
\hline & 14 & $0.2 \%$ & 1 & $<0.0 \%$ & .070 \\
\hline \multicolumn{6}{|l|}{ Incision/wound complications } \\
\hline Incisional hernia & 10 & $0.1 \%$ & 18 & $0.7 \%$ & $<.001^{\dagger}$ \\
\hline Incisional, back or abdominal pain & 12 & $0.2 \%$ & 9 & $0.4 \%$ & .116 \\
\hline Wound infection & 91 & $1.4 \%$ & 50 & $2.0 \%$ & $.034^{\dagger}$ \\
\hline \multirow[t]{2}{*}{ Other } & 3 & $<0.1 \%$ & 3 & $0.1 \%$ & .218 \\
\hline & 116 & $1.7 \%$ & 80 & $3.1 \%$ & $<.001^{\dagger}$ \\
\hline \multicolumn{6}{|l|}{ Neural complications } \\
\hline Extremity neuropathy & 13 & $0.2 \%$ & 3 & $0.1 \%$ & .430 \\
\hline \multirow[t]{2}{*}{ Paralysis, emesis, vomitus } & 4 & $0.1 \%$ & 2 & $0.1 \%$ & .752 \\
\hline & 17 & $0.3 \%$ & 5 & $0.2 \%$ & .611 \\
\hline \multicolumn{6}{|l|}{ Pulmonary complications } \\
\hline Pneumonia & 13 & $0.2 \%$ & 4 & $0.2 \%$ & .710 \\
\hline \multirow[t]{2}{*}{ Other } & 7 & $0.1 \%$ & 1 & $<0.1 \%$ & .340 \\
\hline & 20 & $0.3 \%$ & 5 & $0.2 \%$ & .397 \\
\hline \multicolumn{6}{|l|}{ Renal/ureteric complications } \\
\hline Urinary retention & 12 & $0.2 \%$ & 19 & $0.7 \%$ & $<.001^{\dagger}$ \\
\hline Urinary tract infection & 12 & $0.2 \%$ & 19 & $0.7 \%$ & $<.001^{\dagger}$ \\
\hline \multirow[t]{2}{*}{ Other } & 5 & $0.1 \%$ & 2 & $0.1 \%$ & .952 \\
\hline & 29 & $0.4 \%$ & 40 & $1.6 \%$ & $<.001^{\dagger}$ \\
\hline \multicolumn{6}{|l|}{ Vascular/hematoma complications } \\
\hline Continued bleeding & 13 & $0.2 \%$ & 0 & $0.0 \%$ & $.026^{\dagger}$ \\
\hline Hematoma & 28 & $0.4 \%$ & 8 & $0.3 \%$ & .473 \\
\hline Pulmonary embolism/DVT & 0 & $0.0 \%$ & 5 & $0.2 \%$ & $<.001^{\dagger}$ \\
\hline Subcapsular hematoma & 13 & $0.2 \%$ & 0 & $0.0 \%$ & $.026^{\dagger}$ \\
\hline \multirow[t]{2}{*}{ Other } & 5 & $0.1 \%$ & 6 & $0.2 \%$ & $.045^{\dagger}$ \\
\hline & 59 & $0.9 \%$ & 19 & $0.7 \%$ & .525 \\
\hline Other complications & 135 & $2.0 \%$ & 26 & $1.0 \%$ & $.001^{\dagger}$ \\
\hline Total postoperative complications & 450 & $6.7 \%$ & 220 & $8.6 \%$ & $.002^{\dagger}$ \\
\hline${ }^{\dagger} P<.05$ & & & & & \\
\hline
\end{tabular}

rates of ileus in HALDN donors and re-hospitalization due to ileus. Unexpectedly, post-operative renal and ureteric complications were also significantly elevated among HALDN donors. This may be a concern and may motivate surgeons to select the LDN in preference to the HALDN technique.
However, we found that $50 \%$ of observations came from one HALDN trial [25] and 25\% came from another [29]. Therefore the increased rate of ureteric and renal complications could be due to unidentified center specific practices that are not found at other institutions. 
TABLE 4: Major intraoperative and postoperative complications of LDN and HALDN.

\begin{tabular}{|c|c|c|c|c|c|}
\hline & \multicolumn{2}{|c|}{ LDN } & \multicolumn{2}{|c|}{ HALDN } & $P$-value \\
\hline \multicolumn{6}{|l|}{ Intraoperative complications } \\
\hline \multicolumn{6}{|l|}{ Aborted procedures } \\
\hline Colon injury & 1 & $<0.1 \%$ & 0 & $0.0 \%$ & .537 \\
\hline \multirow[t]{2}{*}{ Mesenteric vein injury } & 1 & $<0.1 \%$ & 0 & $0.0 \%$ & .537 \\
\hline & 2 & $0.0 \%$ & 0 & $0.0 \%$ & .383 \\
\hline Blood transfusions & 51 & $0.8 \%$ & 6 & $0.2 \%$ & $.004^{\dagger}$ \\
\hline \multicolumn{6}{|l|}{ Conversions } \\
\hline Bleeding & 55 & $0.8 \%$ & 11 & $0.4 \%$ & $.047^{\dagger}$ \\
\hline Elective/obesity & 15 & $0.2 \%$ & 4 & $0.2 \%$ & 0.525 \\
\hline \multirow[t]{2}{*}{ Reason not reported/other } & 19 & $0.3 \%$ & 5 & $0.2 \%$ & 0.460 \\
\hline & 89 & $1.3 \%$ & 20 & $0.8 \%$ & $.030^{\dagger}$ \\
\hline \multicolumn{6}{|l|}{ Other } \\
\hline Bowel resection & 1 & $<0.1 \%$ & 0 & $0.0 \%$ & 0.537 \\
\hline \multirow[t]{2}{*}{ Splenectomy } & 4 & $0.1 \%$ & 2 & $0.1 \%$ & 0.752 \\
\hline & 5 & $0.1 \%$ & 2 & $0.1 \%$ & 0.952 \\
\hline Total intraoperative complications & 147 & $2.2 \%$ & 28 & $1.1 \%$ & $.001^{\dagger}$ \\
\hline \multicolumn{6}{|l|}{ Post-operative complications } \\
\hline \multicolumn{6}{|l|}{ Re-hospitalizations } \\
\hline Ileus & 3 & $<0.1 \%$ & 5 & $0.2 \%$ & $.027^{\dagger}$ \\
\hline Infection & 1 & $<0.1 \%$ & 1 & $<0.1 \%$ & .478 \\
\hline \multirow[t]{2}{*}{ Pain } & 0 & $0.0 \%$ & 1 & $0.0 \%$ & .105 \\
\hline & 4 & $0.1 \%$ & 7 & $0.3 \%$ & $.007^{\dagger}$ \\
\hline \multicolumn{6}{|l|}{ Re-operations } \\
\hline Bleeding/hematoma & 10 & $0.1 \%$ & 1 & $<0.1 \%$ & .170 \\
\hline Bowel injury & 2 & $<0.1 \%$ & 1 & $<0.1 \%$ & .823 \\
\hline Incisional hernia & 4 & $0.1 \%$ & 0 & $0.0 \%$ & .217 \\
\hline Small bowel obstruction & 6 & $0.1 \%$ & 3 & $0.1 \%$ & .698 \\
\hline \multirow[t]{2}{*}{ Other } & 4 & $0.1 \%$ & 4 & $0.2 \%$ & .155 \\
\hline & 26 & $0.4 \%$ & 9 & $0.4 \%$ & .807 \\
\hline \multicolumn{6}{|l|}{ Death } \\
\hline Myocardial infarction & 1 & $<0.1 \%$ & 0 & $0.0 \%$ & .537 \\
\hline \multirow[t]{2}{*}{ Pulmonary embolism } & 0 & $0.0 \%$ & 1 & $<0.1 \%$ & .105 \\
\hline & 1 & $0.0 \%$ & 1 & $0.0 \%$ & .478 \\
\hline Total post-operative complications & 31 & $0.5 \%$ & 17 & $0.7 \%$ & .222 \\
\hline Total major complications & 178 & $2.6 \%$ & 45 & $1.8 \%$ & $.013^{\dagger}$ \\
\hline${ }^{\dagger} P<.05$ & & & & & \\
\hline
\end{tabular}


There is debate in the literature if one laparoscopic technique is preferential to the other in obese donors. Heimbach et al. found that HALDN was safe in obese donors (BMI>30 $\mathrm{kg} / \mathrm{m}^{2}$ ); however total operative times and intraoperative complications were increased in significantly obese donors [29]. In contrast, Sundaram et al. did not find significantly elevated operative or post-operative complication rates in obese LDN donors [49]. In our limited analysis, we found that obese LDN and HALDN donors had nearly equivalent BMIs of $26.8 \mathrm{~kg} / \mathrm{m}^{2}$ and $27.3 \mathrm{~kg} / \mathrm{m}^{2}$, respectively. We did not identify differential conversion rates secondary to obesity; however, there is insufficient data to draw firm conclusions on this topic.

Total operative time, warm ischemia time, and length of stay are surrogate measures of outcome. Warm ischemia time was the only operative parameter that was significantly different between the LDN and HALDN groups, with shorter WIT reported in HALDN procedures. Investigators have attributed this difference to the increased tactile control in HALDN, leading to faster vessel management and kidney extraction $[3,6,7,13,40]$. We did not find any statistical difference for either total operative time or length of hospital stay between the techniques. The studies that directly compared the latter two variables in LDN and HALDN procedures reported conflicting trends. It was difficult to compare total operative time with confidence because investigators do not always report a uniform end point that would allow a direct a comparison between institutions. Despite this limitation we observed a wide range in total operative times for each technique (LDN 78.4-253 minutes; HALDN 83-283 minutes). This suggests that center-specific practices and/or experiences influence the operative time reported in the literature.

Our data summarizes the rates of complications and operative statistics reported in the peer-reviewed literature of large institutional studies. Therefore, there are limitations to our study. We have no resources to test the validity of the published findings or identify all the center specific variables that determined the reported outcome. We therefore cannot guarantee that the observations calculated from the summated data can be generalized to other transplant centers. Our conclusions are therefore limited to the specific dataset that was analyzed. While the datasets include a large number of patients, there could be a systematic bias associated with restricting our search to published studies. The use of publications with diverse study designs prevented us from using meta-analysis. Thus, we used simple observational outcomes from the published peer-reviewed literature to create a dataset for analysis and did not use a common measure of effect size. Because we were unable to control the effects of all study characteristics, the dataset incorporates several sets of assumptions and conditions. Even though the data set must be interpreted with caution, it provides a large compendium of outcome information as a first step in assessing performance for quality outcome purposes

At present there is no evidence that proves one laparoscopic technique is superior to the other. There are however consistent trends in the data suggesting that intraoperative injuries are more common in LDN patients while postoperative injuries are more common in HALDN donors. Analysis of major donor morbidity differentiates the two techniques. Transfusions and conversion to open procedures from vascular injury are the most common sources of major donor morbidity reported in the literature. These are cited more frequently in LDN procedures. Conversely, there did not appear to be a difference in the overall rate of major postoperative complications. As such, this paper suggests that the HALDN approach has less associated risk of major donor morbidity.

\section{References}

[1] Department of Health and Human Services HRaSA, Healthcare Systems Bureau, Division of Transplantation, Rockville, Md, USA; United Network for Organ Sharing. Annual report of the US Organ Procurement and Transplantation Network and the Scientific Registry of Transplant Recipients: transplant data 1997-2006, 2007.

[2] A. D. Wright, T. A. Will, D. R. Holt, T. M. Turk, and K. T. Perry, "Laparoscopic living donor nephrectomy: a look at current trends and practice patterns at major transplant centers across the United States," Journal of Urology, vol. 179, no. 4, pp. 14881492, 2008.

[3] I. H. Derweesh, D. A. Goldfarb, S. C. Abreu, et al., "Laparoscopic live donor nephrectomy has equivalent early and late renal function outcomes compared with open donor nephrectomy," Urology, vol. 65, no. 5, pp. 862-866, 2005.

[4] M. Giessing, "Laparoscopic living-donor nephrectomy," Nephrology Dialysis Transplantation, vol. 19, supplement 4, pp. IV36-IV40, 2004.

[5] T. G. Nanidis, D. Antcliffe, C. Kokkinos, et al., "Laparoscopic versus open live donor nephrectomy in renal transplantation: a meta-analysis," Annals of Surgery, vol. 247, no. 1, pp. 58-70, 2008.

[6] G. Ruiz-Deya, S. Cheng, E. Palmer, R. Thomas, and D. Slakey, "Open donor, laparoscopic donor and hand assisted laparoscopic donor nephrectomy: a comparison of outcomes," Journal of Urology, vol. 166, no. 4, pp. 1270-1274, 2001.

[7] N. Simforoosh, A. Basiri, A. Tabibi, N. Shakhssalim, and S. M. Hosseini Moghaddam, "Comparison of laparoscopic and open donor nephrectomy: a randomized controlled trial," British Journal of Urology International, vol. 95, no. 6, pp. 851855, 2005.

[8] G. L. Martin, A. I. Guise, J. E. Bernie, V. Bargman, W. Goggins, and C. P. Sundaram, "Laparoscopic donor nephrectomy: effects of learning curve on surgical outcomes," Transplantation Proceedings, vol. 39, no. 1, pp. 27-29, 2007.

[9] E. H. Chin, D. Hazzan, D. M. Herron, et al., "Laparoscopic donor nephrectomy: intraoperative safety, immediate morbidity, and delayed complications with 500 cases," Surgical Endoscopy, vol. 21, no. 4, pp. 521-526, 2007.

[10] S. Maartense, M. Idu, F. J. Bemelman, R. Balm, S. Surachno, and W. A. Bemelman, "Hand-assisted laparoscopic live donor nephrectomy," British Journal of Surgery, vol. 91, no. 3, pp. 344-348, 2004.

[11] R. C. Minnee, W. A. Bemelman, S. Maartense, F. J. Bemelman, D. J. Gouma, and M. M. Idu, "Left or right kidney in handassisted donor nephrectomy? A randomized controlled trial," Transplantation, vol. 85, no. 2, pp. 203-208, 2008. 
[12] C. Kokkinos, T. Nanidis, D. Antcliffe, A. W. Darzi, P. Tekkis, and V. Papalois, "Comparison of laparoscopic versus handassisted live donor nephrectomy," Transplantation, vol. 83, no. 1, pp. 41-47, 2007.

[13] R. El-Galley, N. Hood, C. J. Young, M. Deierhoi, and D. A. Urban, "Donor nephrectomy: a comparison of techniques and results of open, hand assisted and full laparoscopic nephrectomy," Journal of Urology, vol. 171, no. 1, pp. 40-43, 2004.

[14] K. M. Anderson, T. U. Lindler, G. R. Lamberton, P. W. Baron, O. K. Ojogho, and D. D. Baldwin, "Laparoscopic donor nephrectomy: effect of perirenal fat upon donor operative time," Journal of Endourology, vol. 22, no. 10, pp. 2269-2274, 2008.

[15] V. Bargman, C. P. Sundaram, J. Bernie, and W. Goggins, "Randomized trial of laparoscopic donor nephrectomy with and without hand assistance," Journal of Endourology, vol. 20, no. 10, pp. 717-722, 2006.

[16] A. W. Branco, W. Kondo, A. J. Branco Filho, M. A. de George, M. Rangel, and L. C. Stunitz, "A comparison of hand-assisted and pure laparoscopic techniques in live donor nephrectomy," Clinics (Sao Paulo), vol. 63, no. 6, pp. 795-800, 2008.

[17] A. Breda, J. Veale, J. Liao, and P. G. Schulam, "Complications of laparoscopic living donor nephrectomy and their management: the UCLA experience," Urology, vol. 69, no. 1, pp. 49-52, 2007.

[18] P. Chandak, N. Kessaris, B. Challacombe, J. Olsburgh, F. Calder, and N. Mamode, "How safe is hand-assisted laparoscopic donor nephrectomy? Results of 200 live donor nephrectomies by two different techniques," Nephrology Dialysis Transplantation, vol. 24, no. 1, pp. 293-297, 2009.

[19] M. R. Desai, A. P. Ganpule, R. Gupta, and M. Thimmegowda, "Outcome of renal transplantation with multiple versus single renal arteries after laparoscopic live donor nephrectomy: a comparative study," Urology, vol. 69, no. 5, pp. 824-827, 2007.

[20] E. K. Diner, B. Radolinski, J. D. Murdock, and S. R. Ghasemian, "Right laparoscopic donor nephrectomy: the Washington Hospital Center experience," Urology, vol. 68, no. 6, pp. 1175-1177, 2006.

[21] C. J. Dolce, J. E. Keller, K. C. Walters, et al., "Laparoscopic versus open live donor nephrectomy: outcomes analysis of 266 consecutive patients.," Surgical Endoscopy, vol. 23, no. 7, pp. 1564-1568, 2009.

[22] L. F. C. Dols, N. F. Kok, I. P. J. Alwayn, T. C. Tran, W. Weimar, and J. N. M. Ijzermans, "Laparoscopic donor nephrectomy: a plea for the right-sided approach," Transplantation, vol. 87, no. 5, pp. 745-750, 2009.

[23] R. El-Galley, "Novel technique for hand assisted laparoscopic right donor nephrectomy," Journal of Urology, vol. 178, no. 5, pp. 2062-2066, 2007.

[24] H. A. Fettouh, H. A. Raouf, A. Shenoufy, et al., "Laparoscopic donor nephrectomy: single-center experience in Egypt with 400 consecutive cases," Transplantation Proceedings, vol. 39, no. 4, pp. 807-810, 2007.

[25] P. C. Fisher, J. S. Montgomery, W. K. Johnston III, and J. S. Wolf Jr., "200 consecutive hand assisted laparoscopic donor nephrectomies: evolution of operative technique and outcomes," Journal of Urology, vol. 175, no. 4, pp. 1439-1443, 2006.

[26] F. Giron, Y. Baez, A. Nino-Murcia, J. Rodriguez, and S. Salcedo, "Use of nonabsorbable polymer ligaclip in hand-assisted laparoscopic nephrectomy for living donor," Transplantation Proceedings, vol. 40, no. 3, pp. 682-684, 2008.
[27] M. Gupta, P. Singh, D. Dubey, A. Srivastava, R. Kapoor, and A. Kumar, "A comparison of kidney retrieval incisions in laparoscopic transperitoneal donor nephrectomy," Urologia Internationalis, vol. 81, no. 3, pp. 296-300, 2008.

[28] A. Hawasli, R. Berri, A. Meguid, K. Le, and H. Oh, "Total laparoscopic live donor nephrectomy: a 6-year experience," The American Journal of Surgery, vol. 191, no. 3, pp. 325-329, 2006.

[29] J. K. Heimbach, S. J. Taler, M. Prieto, et al., "Obesity in living kidney donors: clinical characteristics and outcomes in the era of laparoscopic donor nephrectomy," American Journal of Transplantation, vol. 5, no. 5, pp. 1057-1064, 2005.

[30] T. L. Husted, M. J. Hanaway, M. J. Thomas, E. S. Woodle, and J. F. Buell, "Laparoscopic right living donor nephrectomy," Transplantation Proceedings, vol. 37, no. 2, pp. 631-632, 2005.

[31] S. C. Jacobs, E. Cho, C. Foster, P. Liao, and S. T. Bartlett, "Laparoscopic donor nephrectomy: the University of Maryland 6-year experience," Journal of Urology, vol. 171, no. 1, pp. 47-51, 2004.

[32] H. Jeon, T. D. Johnston, S. E. Strup, M. Ibrahim, and D. Ranjan, "University of Kentucky experience with laparoscopic live donor nephrectomy using two different techniques," International Surgery, vol. 91, no. 6, pp. 332-335, 2006.

[33] J. E. Keller, C. J. Dolce, D. Griffin, B. T. Heniford, and K. W. Kercher, "Maximizing the donor pool: use of right kidneys and kidneys with multiple arteries for live donor transplantation," Surgical Endoscopy, vol. 23, no. 10, pp. 2327-2331, 2009.

[34] E. Y. Ko, E. P. Castle, P. J. Desai, et al., "Utility of the endovascular stapler for right-sided laparoscopic donor nephrectomy: a 7-year experience at Mayo clinic," Journal of the American College of Surgeons, vol. 207, no. 6, pp. 896-903, 2008.

[35] B. Kocak, T. B. Baker, A. J. Koffron, and J. R. Leventhal, "Laparoscopic living donor nephrectomy: a single-center sequential experience comparing hand-assisted versus standard technique," Urology, vol. 70, no. 6, pp. 1060-1063, 2007.

[36] I.-R. Lai, M.-K. Tsai, and P.-H. Lee, "Hand-assisted versus total laparoscopic live donor nephrectomy," Journal of the Formosan Medical Association, vol. 103, no. 10, pp. 749-753, 2004.

[37] C. D. Lallas, E. P. Castle, and P. E. Andrews, "Hand port use for extraction during laparoscopic donor nephrectomy," Urology, vol. 67, no. 4, pp. 706-708, 2006.

[38] J. Li, G. Wang, T. Zhu, L. Sun, M. Xu, and R. Rong, "Handassisted laparoscopic donor nephrectomy: a comparative study with conventional open donor nephrectomy in a single Chinese center," Transplantation Proceedings, vol. 40, no. 10, pp. 3362-3364, 2008.

[39] M. L. Melcher, J. T. Carter, A. Posselt, et al., "More than 500 consecutive laparoscopic donor nephrectomies without conversion or repeated surgery," Archives of Surgery, vol. 140, no. 9, pp. 835-840, 2005.

[40] R. C. Minnee, F. Bemelman, C. Kox, et al., "Comparison of hand-assisted laparoscopic and open donor nephrectomy in living donors," International Journal of Urology, vol. 15, no. 3, pp. 206-209, 2008.

[41] L. S. Percegona, A. T. Bignelli, A. Adamy Jr., et al., "Handassisted laparoscopic donor nephrectomy: comparison to pure laparoscopic donor nephrectomy," Transplantation Proceedings, vol. 40, no. 3, pp. 687-688, 2008.

[42] S. Permpongkosol, R. E. Link, L.-M. Su, et al., "Complications of 2,775 urological laparoscopic procedures: 1993 to 2005," Journal of Urology, vol. 177, no. 2, pp. 580-585, 2007.

[43] A. Rajab, J. E. Mahoney, M. L. Henry, et al., "Hand-assisted laparoscopic versus open nephrectomies in living donors," Canadian Journal of Surgery, vol. 48, no. 2, pp. 123-130, 2005. 
[44] R. Ruszat, T. Sulser, M. Dickenmann, et al., "Retroperitoneoscopic donor nephrectomy: donor outcome and complication rate in comparison with three different techniques," World Journal of Urology, vol. 24, no. 1, pp. 113-117, 2006.

[45] A. Salazar, R. Pelletier, S. Yilmaz, et al., "Use of a minimally invasive donor nephrectomy program to select technique for live donor nephrectomy," The American Journal of Surgery, vol. 189, no. 5, pp. 558-563, 2005.

[46] I. S. Seo, J. C. Kim, K. Hwangbo, Y. H. Park, and T. K. Hwang, "Comparison of hand-assisted laparoscopic and open donor nephrectomy: a single-center experience from South Korea," Journal of Endourology, vol. 19, no. 1, pp. 58-62, 2005.

[47] N. Simforoosh, A. Aminsharifi, S. Zand, and A. Javaherforooshzadeh, "How to improve the safety of polymer clips for vascular control during laparoscopic donor nephrectomy," Journal of Endourology, vol. 21, no. 11, pp. 1319-1322, 2007.

[48] L.-M. Su, L. E. Ratner, R. A. Montgomery, et al., "Laparoscopic live donor nephrectomy: trends in donor and recipient morbidity following 381 consecutive cases," Annals of Surgery, vol. 240, no. 2, pp. 358-363, 2004.

[49] C. P. Sundaram, G. L. Martin, A. Guise, et al., "Complications after a 5-year experience with laparoscopic donor nephrectomy: the Indiana University experience," Surgical Endoscopy, vol. 21, no. 5, pp. 724-728, 2007.

[50] D. A. Duchene and H. N. Winfield, "Laparoscopic donor nephrectomy," Urologic Clinics of North America, vol. 35, no. 3, pp. 415-424, 2008. 


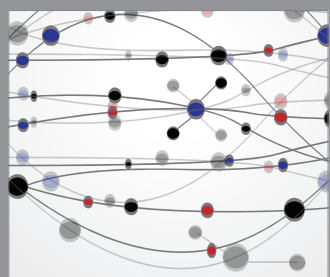

The Scientific World Journal
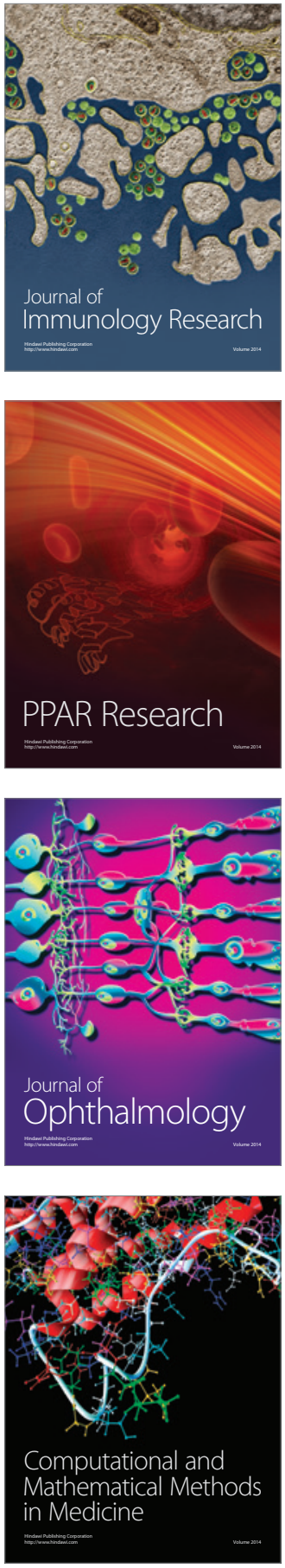

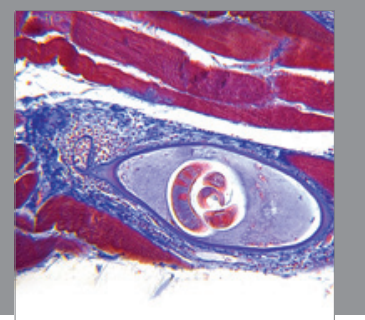

Gastroenterology

Research and Practice
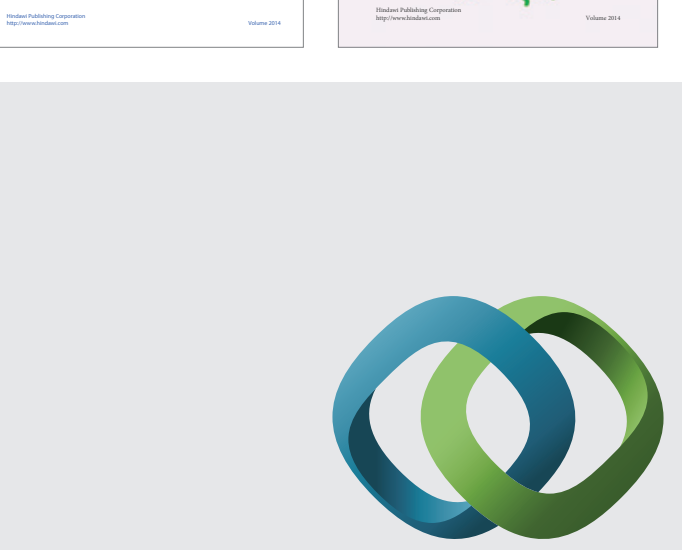

\section{Hindawi}

Submit your manuscripts at

http://www.hindawi.com
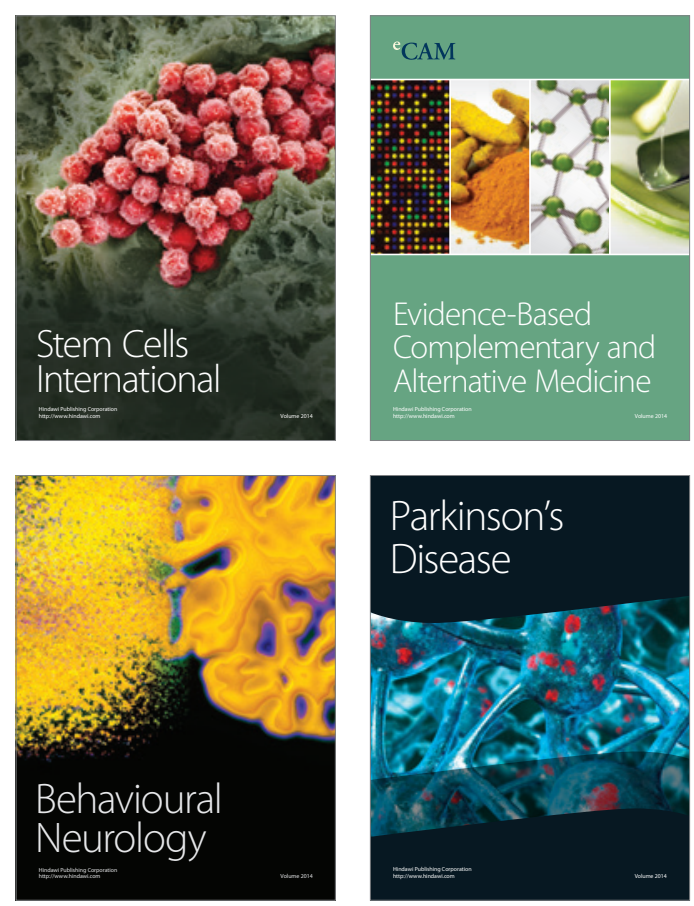

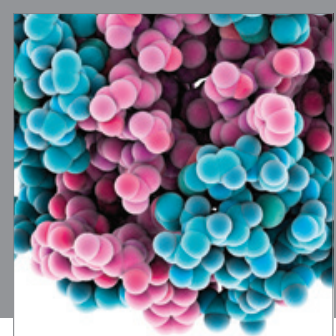

Journal of
Diabetes Research

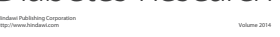

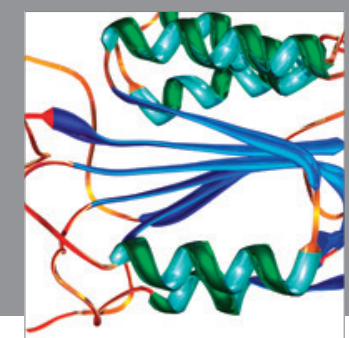

Disease Markers
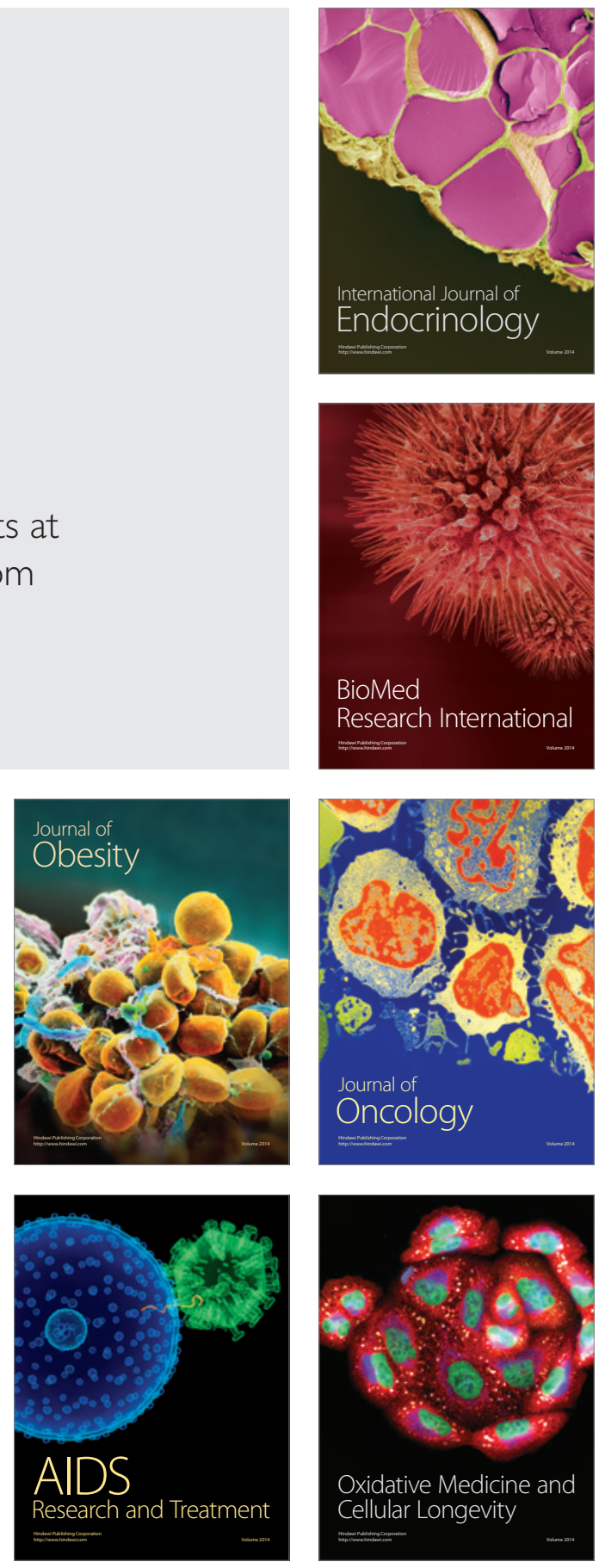